\title{
AN EFFECTIVE APPROACH OF APPROXIMATION OF FRACTIONAL ORDER SYSTEM USING REAL INTERPOLATION METHOD
}

\author{
Quang Dung NGUYEN* \\ Faculty of Electrical and Electronics Engineering, Ton Duc Thang University, Ho Chi Minh City, \\ Vietnam \\ *nguyenquangdung@tdt.edu.vn \\ (Received: 11-February-2017; accepted: 30-April-2017; published: 8-June-2017) \\ DOI: http://dx.doi.org/10.25073/jaec.201711.48
}

\begin{abstract}
Fractional-order controllers are recognized to guarantee better closed-loop performance and robustness than conventional integerorder controllers. However, fractional-order transfer functions make time, frequency domain analysis and simulation significantly difficult. In practice, the popular way to overcome these difficulties is linearization of the fractional-order system. Here, a systematic approach is proposed for linearizing the transfer function of fractionalorder systems. This approach is based on the real interpolation method (RIM) to approximate fractional-order transfer function (FOTF) by rational-order transfer function. The proposed method is implemented and compared to CFE high-frequency method; Carlson's method; Matsuda's method; Chareff's method; Oustaloup's method; least-squares, frequency interpolation method (FIM). The results of comparison show that, the method is simple, computationally efficient, flexible, and more accurate in time domain than the above considered methods.
\end{abstract}

\section{Keywords}

Approximation, fractional-order system, real interpolation method.

\section{Introduction}

The concept of fractional calculus has appeared long time ago but due to its complexity, it could not be used in many applications. It is only in the recent years with rapid development of hardware and software applications in computer and electronics fields that fractional calculus theory has been widely used in many applications of science and engineering, including acoustics [1], 2], robotics [3], 4], biomedical engineering, control systems [5], [6], [7] and signal processing [8], 9]. In fact, one could argue that real world processes are fractional order systems in general [10, [11].

Fractional-order models are infinite dimensional, and more adequate for the description of dynamical systems than the integer-order models. In technical literature, fractional-order differential equations are mostly analyzed using Laplace transform techniques [10. However, the signals involved in these applications are characterized by irrational Laplace transform, so that the inverse transforms are generally not easily evaluated and the time-domain analysis faces a lot of difficulties.

As mentioned above, one of the major difficulties with fractional order representation is the computation of frequency, and especially time responses. Many studies have been done in order to simulate fractional control systems over the last decade. The analytical solution of the 
output is not practical and there is no a general method for estimating it [12]. There are also some methods based on Mittag-Leffler functions, Grunwald-Letnikov fractional derivative and Gamma functions for computation of the impulse and step responses of commensurateorder system [13, [14]. However, the solution methods using Mittag-Leffler functions and Gamma function are time consuming and highly inaccurate, occurring in solving complicated and high fractional-order differential equation.

One possible approach to modelling fractional order system is based on numerical approximation of the non-integer order operator [15, [16, 17]. The methods developing integer order approximations are attractive since, they convert the problems related to the FOTFs into classical transfer functions. Therefore a large number of methods to evaluate rational approximations have been developed. The most popular of these are listed: frequency interpolations, continued fractional expansion (CFE) method, Oustaloup's method, Carlson's method, Matsuda's method, Chareff's method, and leastsquare method.

The approximation methods in frequency domain are represented as frequency interpolation methods (FIM) [18. These methods require separating real and imaginary parts of the fractional order transfer function when replacing the frequency variables. The approximation results could have high accuracy in frequency domain. However, in time domain, accuracy is uncertain especially with low approximated order function.

Some studies are based on a continued fractions expansion (CFE) [19], or modified CFE such as Carlson method [20], 21]. Many researchers have been working in this area and have been successful in developing some approximation techniques, applied to the frequency variables. These are Matsuda method [16], Chareff's method [15], Oustaloup's method [22, [23] and the method proposed by Xue et al [24]. These methods produce approximated integer order models whose characteristics fit closely enough to the ideal system characteristics in the desired frequency bandwidth. Out of these, some methods approximate very high integer-order models for attaining desired accuracy in the desired frequency ranges. In such cases, a reduced order model can be required from a high integer order transfer function [24].

Most of the approximation methods are studied in the frequency domain, because of their accuracy in the time domain might not reach the desired value. This paper introduces an approach for inverting the transfer function of fractional-order systems to rational transfer function with commensurate order. The proposed approach is based on the real interpolation method [25], [26, which is characterized by two main features. The first feature involves the operator method, in which the problem is solved in the imaginary domain, where computation has certainly more advantages than in the time domain. The second feature is that the models in the RIM are a function of a real variable, comparing with a model producing in the imaginary domain or in the complex domain

\section{Real Interpolation Method}

RIM is one of the methods, which works on mathematical descriptions of the imaginary domain. The method is based on real integral transform,

$$
F(\delta)=\int_{0}^{\infty} f(t) e^{-\delta \cdot t} d t, \delta \in(C, \infty), C \geq 0
$$

which assigns the image function $F(\delta)$ in accordance to the original function $f(t)$ as a function of the real variable $\delta$. Formula of direct transform can be considered as a special case of the direct Laplace transform by replacing the complex variable $s$ for real $\delta$ variable. Another step towards the development of the instrumentation method is the transition from continuous functions $F(\delta)$ to their discrete form, using the computing resources and numerical methods. For these purposes, RIM is represented by the numerical characteristics $\left\{F\left(\delta_{i}\right)\right\}_{N}$. They are obtained as a set of values of function $F(\delta)$ in the nodes $\delta_{i}$ where $i \in 1,2, \ldots N$, where $N$ is the 
number of elements of numerical characteristics, called its dimension.

Selecting of interpolations $\delta_{i}$ is a primary step in the transition to a discrete form, which has a significant impact on the numerical computing and accuracy of problem solutions. Distribution of nodes in the simplest variant is uniform. Another important advantage of the RIM is crossconversion property. It dues to the fact that the behavior of the function $F(\delta)$ for large values of the argument $\delta$ is determined mainly by the behavior of the original $f(t)$ for small values of the variable $t$. In the opposite case, the result is the same: the behavior of the function $F(\delta)$ for small values of the argument $\delta$ is determined mainly by the behavior of the original $f(t)$ for large values of the variable $t$.

\section{Rational}

Approximation of FOTs Using Real

\section{Interpolation Method}

In this paper we consider the following approximation task of fractional-order systems. The FOTF is given by the following expression:

$$
G(s)=\frac{K(s)}{L(s)}=\frac{\sum_{i}^{p} k_{i} s^{\beta_{i}}}{\sum_{i}^{q} l_{i} s^{\alpha_{i}}},
$$

where $\quad p, q-$ interger and $\beta_{i}, \alpha_{i}-$ real numbers.

Let us consider rational transfer function:

$$
W(s)=\frac{B(s)}{A(s)}=\frac{b_{m} s^{m}+\cdots+b_{1} s+b_{0}}{a_{n} s^{n}+\cdots+a_{1} s+a_{0}},
$$

where $m \leq n ; m, n$ are the integer, which should be used to approximate transfer function $G(s)$ of linear fractional order system. For $\left(G(0) \neq 0, b_{0}=1\right)$ or $\left(G(0)=0, a_{0}=1\right)$ there are $N=n+m+1$ real coefficients which should be determined from $N$ equations obtained from the condition of overlapping the numerical characteristics in the corresponding discrete points,

$$
\begin{gathered}
G\left(\delta_{i}\right)-\frac{B\left(\delta_{i}\right)}{A\left(\delta_{i}\right)}=0, i=\overline{1, N}, \\
G\left(\delta_{i}\right) A\left(\delta_{i}\right)-B\left(\delta_{i}\right)=0, \quad i=\overline{1, N},
\end{gathered}
$$

or for $G(0)=0, a_{0}=1$ one obtained

$$
\begin{gathered}
a_{n} \delta_{i}^{n} G\left(\delta_{1}\right)+\ldots+a_{1} \delta_{i} G\left(\delta_{i}\right)-b_{m} \delta_{i}^{m}- \\
-b_{0}=-G\left(\delta_{i}\right), i=\overline{1, N},
\end{gathered}
$$

For fixed $\delta_{i}$ both numerator and denominator polynomials are linear combinations of the unknown process parameters. Thus, the set of equations (9) represents a linear system of equations having $\mathrm{N}$ linear equations, one obtains $\mathrm{N}$ coefficients of the rational approximation Eq. 3.

The obtained Eq. 5 are conveniently rewritten in the following matrix form, which is easily solved using some of the modern computer algebra packages, in particular, introducing

$$
\begin{aligned}
& M= \\
& {\left[\begin{array}{ccc}
\delta_{N, 1}^{n} G\left(\delta_{N, 1}\right) \ldots & \delta_{N-n, 1} G\left(\delta_{N-n, 1}\right)-\delta_{N-n-1,1}^{m} \ldots & -1 \\
\delta_{N, 2}^{n} G\left(\delta_{N, 2}\right) \ldots & \delta_{N-n, 2} G\left(\delta_{N-n, 2}\right)-\delta_{N-n-1,2}^{m} \cdots & -1 \\
\delta_{N, N}^{n} G\left(\delta_{N, N}\right) \ldots & \delta_{N-n, N} G\left(\delta_{N-n, N}\right)-\delta_{N-n-1, N}^{m} \ldots & -1
\end{array}\right],} \\
& B=\left[\begin{array}{c}
-G\left(\delta_{1}\right) \\
-G\left(\delta_{2}\right) \\
\ldots \\
-G\left(\delta_{N}\right)
\end{array}\right]
\end{aligned}
$$

one easily obtains the desired system of linear equations in matrix form

$$
M \cdot X=B,
$$

where $\mathrm{X}$ is the vector of unknown parameters,

$$
X=\left[\begin{array}{c}
a_{n} \\
a_{n-1} \\
\cdots \\
a_{1} \\
b_{m} \\
\cdots \\
b_{0}
\end{array}\right]
$$

It is important to mention that the selected set of points $\delta \in\left[\delta_{1}, \delta_{2}, . ., \delta_{N}\right]$ can produce a singular matrix from the set of equations. In such a case, another, more appropriate set of points should be used. It is also significant to note that it is also possible to use more than $n$ incident points in the selected set. The exact solution cannot be found in such a case. 


\section{Numerical Examples and Discussion}

RIM is one of the methods, which works on mathematical descriptions of the imaginary domain. The method is based on real integral transform,

Let us select several FOTFs and compare their Bode characteristics and response to Heaviside excitation with those of the corresponding rational approximation determined on the basis of the set of linear equations Eq. 5. In these examples there are comparisons between the Heaviside responses, Bode characteristics of the approximation models and the exact model.

The following example demonstrates a case when the process outputs is equal to the fractional capacitor. The actual transfer function has a form: $G_{1}(s)=1 / s^{0.5}$.

For transfer function of the fractional capacitor with fractional order 0.5 we carry out approximation using RIM and compare to different methods CFE high-frequency method; Matsuda's method; Carlson's method; least-squares method then estimate the adequacy of approximation models 12 .

According to considered model Eq. $6 G_{1}(0) \rightarrow$ $\infty$ and $G_{1}(\infty) \rightarrow 0$, we choose approximation model Eq. 3 with $b_{0}=1, a_{0}=0$. The orders of the approximated rational transfer function being considered are $n=3$ in numerator, and $m=4$ in denominator. It means that number of unknown coefficients is $N=n+m=7$. Corresponding to $4^{\text {th }}$ order of the ration approximated transfer function, the number of unknown coefficient is $N=7$. In the RIM we choose values of the nodes $\delta_{i}$ in range the $[0.001 ; 0.005 ; 0.01 ; 0.05 ; 0.1 ; 1 ; 5]$ with $N=7$ nodes equally spaced. The results of approximation process will be analysed in the time and frequency domains and compared to other methods with same order of approximation model.

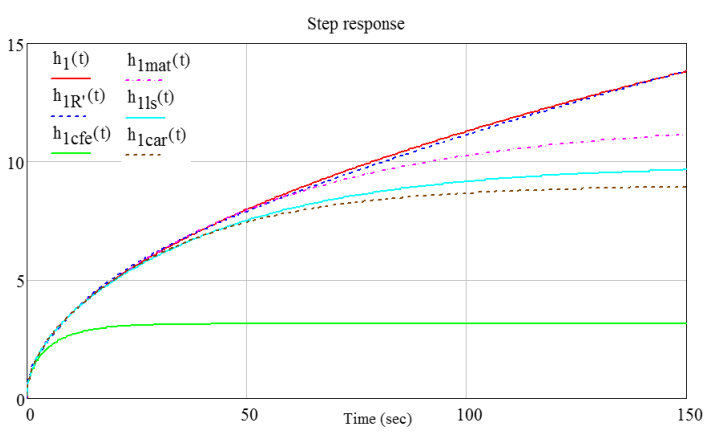

Fig. 1: Time responses

where $\mathrm{h}_{1}(\mathrm{t})$ - exact time response; $\mathrm{h}_{1 R^{\prime}}(\mathrm{t})$ - time response by RIM method; $\mathrm{h}_{1 c f e}(\mathrm{t})$ - time response by CFE method; $h_{1 m a t}(\mathrm{t})$ - time response by Matsuda's method; $\mathrm{h}_{1 l s}(\mathrm{t})$ - time response Least-squares method; $h_{1 \text { car }}(\mathrm{t})$ - time response by Carlson's method.

The approximation errors of time responses are illustrated in Fig. 2 ,

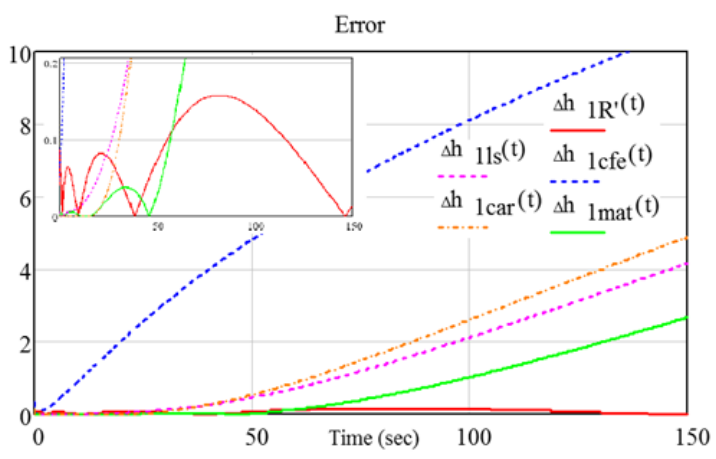

Fig. 2: Approximation error of time responses

where $\Delta \mathrm{h}_{1 R^{c}}(\mathrm{t})$ - error of time response by RIM method; $\Delta \mathrm{h}_{1 c f e}(\mathrm{t})$ - error of time response by CFE method; $\Delta \mathrm{h}_{1 \text { mat }}(\mathrm{t})$ - error of time response by Matsuda's method; $\Delta \mathrm{h}_{1 l s}(\mathrm{t})$ - error of time response Least-squares method; $\Delta \mathrm{h}_{1 c a r}(\mathrm{t})$ - error of time response by Carlson's method.

Tab. 1: Maximum approximation error in time range [0-150] (sec).

\begin{tabular}{|l|c|c|c|c|c|}
\hline & RIM & CFE & $\begin{array}{c}\text { Matsuda's } \\
\text { method }\end{array}$ & $\begin{array}{c}\text { LS- } \\
\text { method }\end{array}$ & $\begin{array}{c}\text { Carlson' } \\
\text { method }\end{array}$ \\
\hline Error & 0.158 & 10.66 & 2.674 & 4.168 & 4.889 \\
\hline
\end{tabular}


According to the time responses of the approximated rational transfer function, the time response of the transfer function approximated by the RIM demonstrates significantly higher accuracy than the other methods in considered range of time. Maximum approximation errors in Table 1. show that maximum approximation of the RIM model is lower than Matsuda's method, having second place and CFE method, having least accuracy in the error about 17 and 67 times, respectively.

The Bode plots of the approximation models are shown in the below figures. The Bode plots illustrate the logarithmic magnitude, phase responses, and errors plots, respectively.

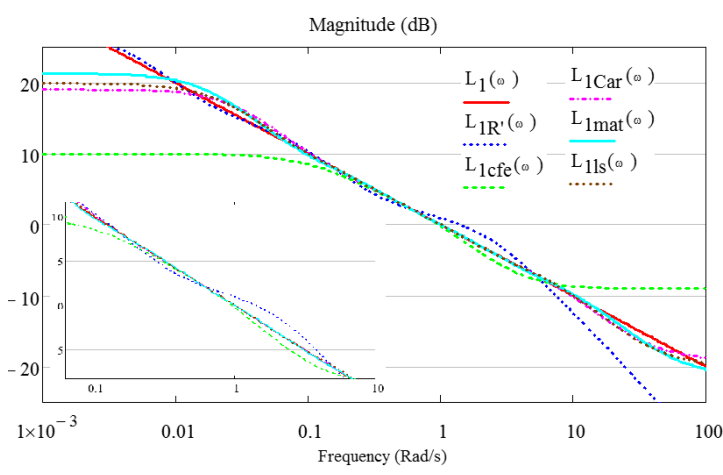

Fig. 3: Magnitude responses

where: $\mathrm{L}_{1}(\omega)$ - exact magnitude response; $\mathrm{L}_{1 R^{\prime}}(\omega)$ - magnitude response by RIM method; $\mathrm{L}_{1 c f e}(\omega)$ - magnitude response by CFE method; $\mathrm{L}_{1 \text { mat }}(\omega)$ - magnitude response by Matsuda's method; $\mathrm{L}_{1 l s}(\omega)$ - magnitude response by leastsquares method; $\mathrm{L}_{1 \text { car }}(\omega)$ - magnitude response by Carlson's method.

where: $\Delta \mathrm{L}_{1 R^{\prime}}(\omega)$ - error of magnitude response by RIM method; $\Delta \mathrm{L}_{1 c f e}(\omega)$ - error of magnitude response by $\mathrm{CFE}$ method; $\Delta \mathrm{L}_{1 \text { mat }}(\omega)$ - error of magnitude response by Matsuda's method; $\Delta \mathrm{L}_{1 l s}(\omega)$ - error of magnitude response by leastsquares method; $\Delta \mathrm{L}_{1 \text { car }}(\omega)$ - error of magnitude response by Carlson's method.

where: $\operatorname{Arg}_{1}(\omega)$ - exact phase response; $\operatorname{Arg}_{1 c f e}(\omega)$ - phase response by CFE method; $\operatorname{Arg}_{1 m a t}(\omega)$ - phase response by Matsuda's method; $\operatorname{Arg}_{1 l s}(\omega)$ - phase response by leastsquares method; $\operatorname{Arg}_{1 R^{\prime}}(\omega)$ - phase response by

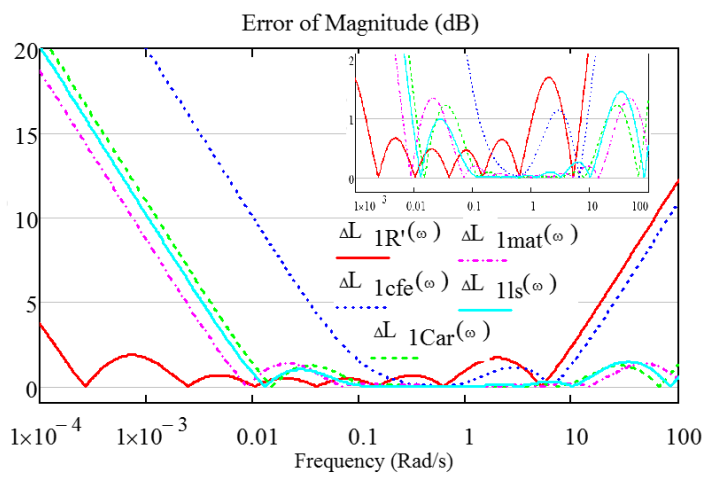

Fig. 4: Errors of the magnitude responses

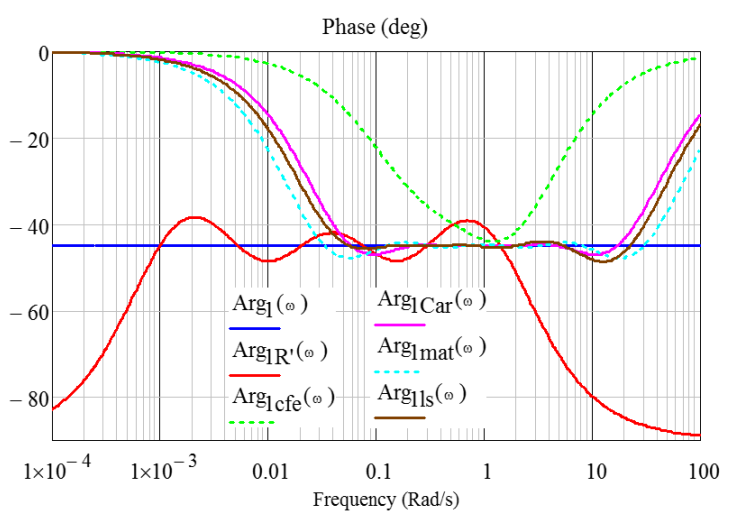

Fig. 5: Phase responses

RIM method; $\operatorname{Arg}_{1 c a r}(\omega)$ - phase response by Carlson's method.

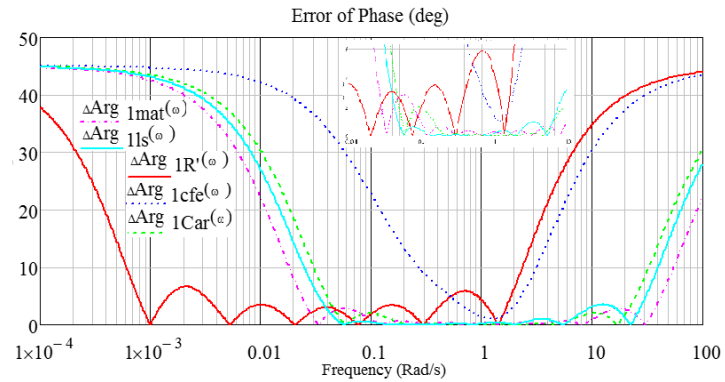

Fig. 6: Errors of the phase responses

where: $\Delta \operatorname{Arg}_{1 c f e}(\omega)$ - error of phase response by CFE method; $\Delta \operatorname{Arg}_{1 m a t}(\omega)$ - error of phase response by Matsuda's method; $\Delta \operatorname{Arg}_{1 l s}(\omega)$ - error of phase response by least-squares method; $\Delta \operatorname{Arg}_{1 R^{\prime}}(\omega)$ - error of phase response by RIM method; $\Delta \operatorname{Arg}_{1 \text { car }}(\omega)$ - error of phase response by Carlson's method. 
Fig. 4 and Fig. 6 show that the errors in magnitude and phase responses of the considered approximation methods present the lowest value in low frequency ranged $\left[10^{-3}, 0.1\right] \mathrm{Hz}$. In higher and lower frequency regime, results of the RIM introduce less accuracy. Generally the RIM in Bode diagrams fit the exact model in the wide range comparing to the other methods.

Second part of example we compare RIM to approximation methods: Chareff's method; Oustaloup's method and frequency interpolation method. The orders of the approximated rational transfer function are chosen higher the previous example with $n=4$ in numerator and $m=5$ in denominator. It means that number of unknown coefficients is $N=n+$ $m=9$ with $a_{0}=0$ and $b_{0}=1$. In the RIM we choose values of the nodes $\delta_{i}$ in range $[0.001 ; 0.005 ; 0.01 ; 0.05 ; 0.1 ; 1 ; 5 ; 10 ; 50]$ with $N=9$ nodes equally spaced. The results of approximation process will be considered in the time and frequency domains.

The exact time response of the fractional order system, as well as those of the approximation models, are presented in Fig. 7. In additional approximation errors are illustrated in Fig. 8.

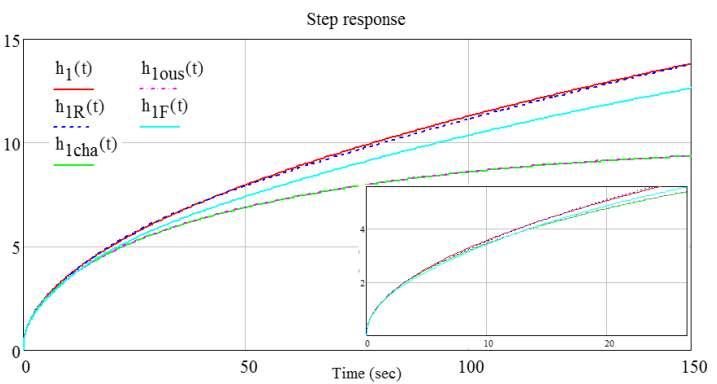

Fig. 7: Time responses

where: $\mathrm{h}_{1}(\mathrm{t})$ - exact time response; $\mathrm{h}_{1 R^{\prime}}(\mathrm{t})$ - time response by RIM method; $\mathrm{h}_{1 \text { cha }}(\mathrm{t})$ - time response by Chareff's method; $\mathrm{h}_{\text {lous }}(\mathrm{t})$ - time response by Oustaloup's method; $\mathrm{h}_{1 F}(\mathrm{t})$-time response by FIM.

where $\Delta \mathrm{h}_{1 R^{\prime}}(\mathrm{t})$ - error of time response by RIM method; $\Delta \mathrm{h}_{1 \text { cha }}(\mathrm{t})$ - error of time response by Charref's method; $\Delta \mathrm{h}_{1 \text { ous }}(\mathrm{t})$ - error of time response by Oustaloup's method; $\Delta \mathrm{h}_{1 F}(\mathrm{t})$ - error of time response by FIM.

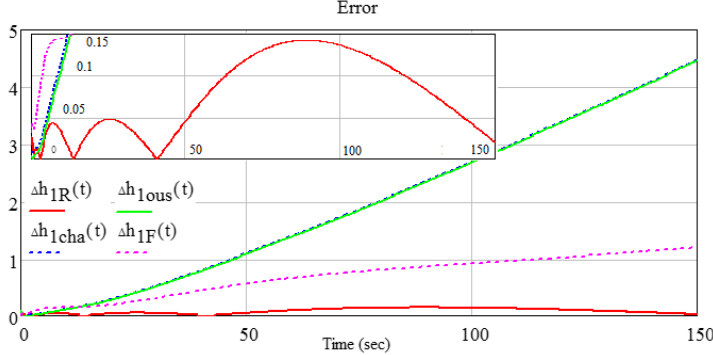

Fig. 8: Approximation error of time responses

According to the Fig. 8, the maximum approximations can be determined and are listed in Tab. 2.

Tab. 2: Maximum approximation error in time range [0-150] (sec).

\begin{tabular}{|l|c|c|c|c|}
\hline & RIM & $\begin{array}{c}\text { Cha. } \\
\text { Method }\end{array}$ & $\begin{array}{c}\text { Oustaloup's } \\
\text { method }\end{array}$ & FIM \\
\hline Error & 0.142 & 4.467 & 4.453 & 1.193 \\
\hline
\end{tabular}

As presented in the Fig. 7 and Fig.9 it clearly shows that, new method provides a well-fitting. Comparing Fig. 2 and Fig. 8 detailed in Tab. 1 and Tab.2 2 it leads to the conclusion that error of $4^{\text {th }}$ order model (about 0.158 ) is higher than $5^{\text {th }}$ order model, approximated (about 0.142 ) by RIM. Consequently, the $5^{\text {th }}$ order models are more accurate than the $4^{\text {th }}$ order model, approximated by RIM.

The Bode plots of the approximation models are shown in the Fig. 9 12,

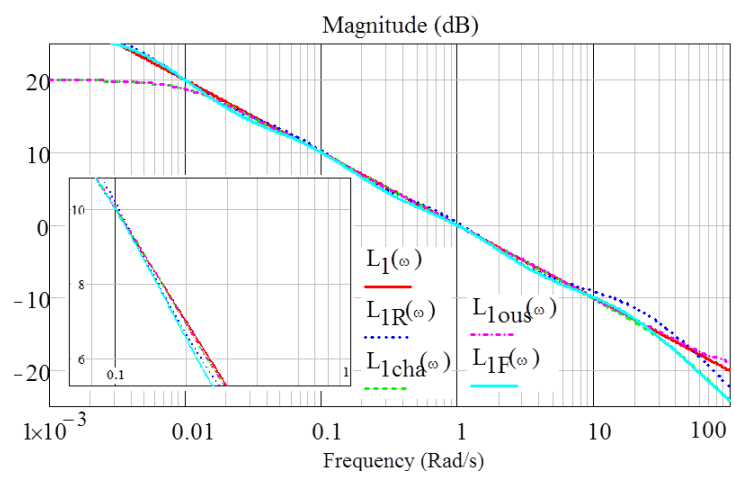

Fig. 9: Magnitude responses

where: $\mathrm{L}_{1}(\omega)$ - exact magnitude response; $\mathrm{L}_{1 R}(\omega)$ - magnitude response by RIM method; $\mathrm{L}_{1 \text { cha }}(\omega)$ - magnitude response by Charref's 
method, $\mathrm{L}_{1 \text { ous }}(\omega)$ - magnitude response by Oustaloup's method; $\mathrm{L}_{1 F}(\omega)$ - magnitude response by FIM.

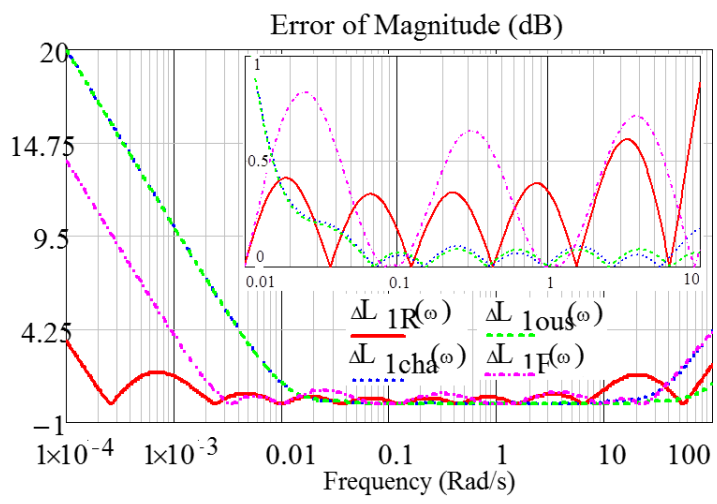

Fig. 10: Errors of the magnitude responses

where: $\Delta \mathrm{L}_{1 R}(\omega)$ - magnitude response error by RIM method; $\Delta \mathrm{L}_{1 \text { cha }}(\omega)$ - magnitude response error by Charref's method; $\Delta \mathrm{L}_{1 \text { ous }}(\omega)$ - magnitude response error by Oustaloup's method; $\Delta \mathrm{L}_{1 F}(\omega)$ - magnitude response error by FIM.

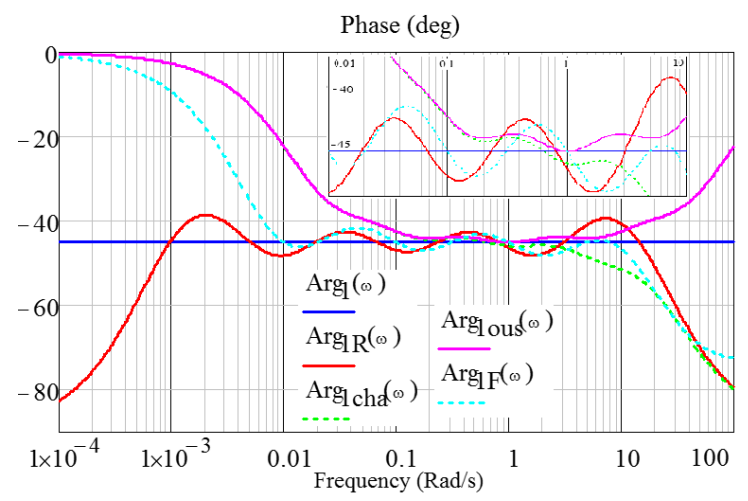

Fig. 11: Phase responses

where: $\operatorname{Arg}_{1}(\omega)$ - exact phase response; $\operatorname{Arg}_{1 R}(\omega)$ - phase response by RIM method; $\operatorname{Arg}_{1 \text { cha }}(\omega)$ - phase response by Charref's method; $\operatorname{Arg}_{1 \text { ous }}(\omega)$ - phase response by Oustaloup's method; $\operatorname{Arg}_{1 F}(\omega)$ - phase response by FIM.

where: $\Delta \operatorname{Arg}_{1 R}(\omega)$ - phase response error by RIM method; $\Delta \operatorname{Arg}_{1 c h a}(\omega)$ - phase response error by Charref's method; $\Delta \operatorname{Arg}_{1 o u s}(\omega)$ phase response error by Oustaloup's method; $\Delta \operatorname{Arg}_{1 F}(\omega)$ - phase response error by FIM.

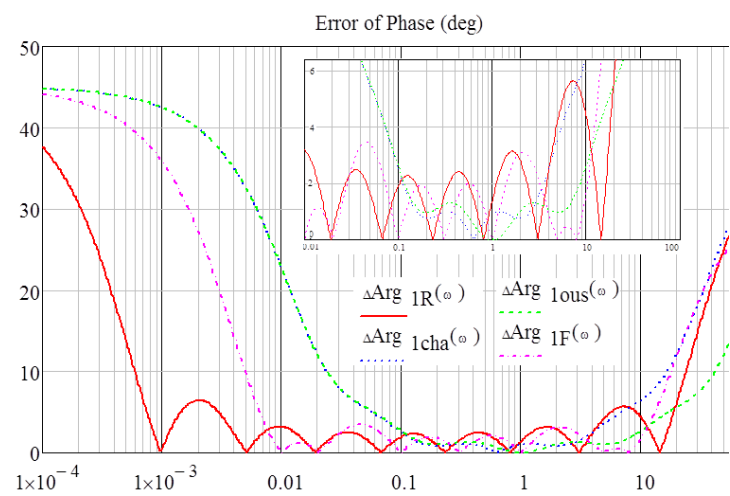

Fig. 12: Errors of the phase responses

The diagrams show that it leads to the same above conclusion, the fitness of RIM model in Bode characteristics is lower than the other methods in the range [0.1-5] Hz. However RIM in Bode diagrams fit the exact model in the wide range about $\left[10^{-3}-10\right] \mathrm{Hz}$. In comparison to $4^{\text {th }}$ order approximation model by RIM, the accuracy of $5^{\text {th }}$ RIM model represents more accurate.

To estimate of the RIM, we carry out numerical examples with typical fractional-order integrator systems, which is monotonic unstable. In the examples, there were conducted indepth analysis of the results of several typical approximation methods and the RIM in the time domain and the frequency domain. The above results show that the accuracy of the RIM in the time domain is significantly higher comparing to considered methods. Maximum approximation error of RIM model is smaller 8.5 times and 67 times comparing to FIM model and CFE model, respectively. The $5^{\text {th }}$ order models is more fitting than the $4^{\text {th }}$ order model, approximated by RIM, is about $10 \%$ more accurate. In the frequency domain as the Bode characteristics, the RIM models show higher fitting than considered methods in low and high ranges of the considered frequencies. However, near the medium-frequency range of the considered frequency range, the RIM is less satisfactory than other methods. Generally the RIM in Bode diagrams fit the exact model in the wide range comparing to the other methods 


\section{Conclusion}

In this paper, a new approximation method for fractional-order system is presented. The most significant feature of the proposed method is its computational efficiency. Another advantage of the RIM is its high accuracy in the time domain, comparing to the conventional methods. The higher order RIM models are more accurate then the lower order RIM models. In fact, this method is very simple both conceptually and computationally. The obtained results from the previous examples are quite satisfactory. The main drawback of the proposed method is that it is uncertain of the approximation model in the frequency domain. Another limitation of the method is not possible to guarantee the stability a priori, in other words no constraints on the coefficients are enforced. Indeed, the form of these constraint would be so complicated, so that their introduction would impair the established efficiency of the solution presented in the current paper.

\section{References}

[1] BARBU, M., Edit Kaminsky and R. E. Trahan "Acoustic Seabed Classification using Fractional Fourier Transform and TimeFrequency Transform Techniques", in Proceedings of the IEEE Oceanic Engineering Society. Boston, US, pp. 45-51, 2006.

[2] BARBU, M., Edit Kaminsky, and R. E. Trahan, "Fractional Fourier transform for sonar signal processing', in the IEEE Oceanic Engineering Society. Washington DC, US, 2005.

[3] KESARKAR, A. A. and S. NARAYANASAMY. "Investigation on Superior Performance by Fractional Controller for Cart-Servo Laboratory Set-Up", Advances in Electrical and Electronic Engineering, vol. 12, iss. 3, pp. 201-209, 2014.

[4] CHEN, H. and Y. Chen, "Fractional-order generalized principle of self-support (FOGPSS) in control system design", Journal of
Automatica Sinica., vol. 3, iss. 4, pp. 430441, 2016.

[5] MOVAHHED, A. M., H. T. SHADIZ and S. K. H. SANI. "Comparison of Fractional Order Modelling and Integer Order Modelling of Fractional Order Buck Converter in Continuous Condition Mode Operation" Advances in Electrical and Electronic Engineering, vol. 14, iss. 5, pp. 531-542, 2016.

[6] SIROTA, L., and Y. HALEVI, "Fractional order control of flexible structures governed by the damped wave equation", in Proceeding of the American Control Conference. Chicago, USA, pp. 565-570, 2015.

[7] JOSHI, M. M., V. A. VYAWAHARE and M. D. PATIL. "Model predictive control for fractional-order system a modeling and approximation based analysis", in Proceeding of the 4th International Conference On Simulation And Modeling Methodologies, Technologies And Applications, Vienna, Austria, 2014.

[8] MAIONE, G. "Concerning continued fractions representations of noninteger order digital differentiators", IEEE transaction of Signal Process, vol. 13, iss. 12, pp. $735-$ 738, 2007.

[9] NEZZARI, H., A. CHAREF and D. BOUCHERMA. Analog Circuit Implementation of Fractional Order Damped Sine and Cosine Functions. IEEE Journal on Emerging and Selected topics in Circuits and Systems, vol. 3, iss. 3, pp. $386-393$, 2013.

[10] GONZALEZ, E. A., and I. PETRAS, "Advances in fractional calculus- Control and signal processing applications", in Procceding of the $16^{\text {th }}$ Carpathian Control Conference. Hungary, 2015.

[11] DZIELINSKI, A., D. SIEROCIUK and G. SARWAS. Some applications of fractional order calculus, Automatics, vol. 58, iss. 4, pp. 583-593, 2010.

[12] ATHERTON, D. P., N. TAN and A. YUCE. Methods for computing the time response of 
fractional-order systems, IET Control theory 85 Applications, vol. 9, iss. 6, pp. 817830, 2014.

[13] DORCAK, L., E. A. GONZALEZ, J. TERPAK, J. VALSA and L. PIVKA. Identification of fractional-order dynamical, International Journal of Pure and Applied Mathematics, vol. 89, iss. 2, pp. 335-350, 2013.

[14] REKANOS I. T. and T. V. YIOULTSIS. "Approximation of Grünwald-Letnikov Fractional Derivative for FDTD Modeling of Cole-Cole Media". IEEE Transactions on magnetics, vol. 50, iss. 2, pp. $181-184$, 2014.

[15] OVIVIER, P. D. "Approximating irrational transfer functions using Lagrage interpolation formula", IEE Proceedings D - Control Theory and Applications, vol. 139, iss. 1, pp. 9-13, 1992.

[16] KRISHNA, B. T. "Studies on fractional order differentiators and integrators a survey", Signal Process, vol. 91, iss. 3, pp. 386436, 2011.

[17] DJOUAMBI A., A. CHAREF and A. VODA, "Numerical simulation and identification of fractional systems using digital adjustable fractional order", in Proceeding of the 2013 European control conference. Zurich, Switzerland, 2013

[18] SEKARA, T. B., M. R. RAPAIC and M. P. LAZAREVIC. An Efficient Method for Approximation of Non-Rational Transfer Functions. Electronics. 2013, vol. 17, iss. 1, pp. $40-44$

[19] MAIONE, G. "Continued fractions approximation of the impulse response of fractional-order dynamic systems ", IET Control Theory and Applications, vol. 3, iss. 7, pp. 564-573, 2008.

[20] SHRIVASTAVE, N. and P. VARSHNEY. "Rational approximation of fractional order systems using Carlson method", in Proceeding of the International Conference on Soft Computing Techniques and Implementations. Faridabad, India, pp. 76-80, 2015.
[21] CARLSON, G. E. and C. A. HALIJAH. "Approximation of Fractional Capacitors $(1-s)^{(1-n)}$ by a Regular Newton Process", IEEE Transactions on Circuit Theory," vol. 3, iss. 7, pp. 310-313, 1963.

[22] OUSTALOUP, A., F. LEVRON, B. MATHIEU, F. M. NANOT. "FrequencyBand Complex Noninteger Differentiator: Characterization and Synthesis," IEEE Transactions on Circuit Theory, vol. 47, iss. 7, pp. 25-39, 2000.

[23] KRAJEWSKI, W. and U. VIARO. "A method for the integer-order approximation of fractional-order systems," Journal of the Franklin Institute. 2013, vol. 351, iss. y, pp. 555-564.

[24] XUE, D., Ch. ZHAO and Y. Q. CHEN. "A modified approximation method of Fractional order system," in Proceeding of the 3006 IEEE International Conference on Mechatronics and Automation. Louyang, China, pp. 1043-1048, 2006.

[25] GONCHAROV, V. I. Editors, Real interpolation method in control automation issue. $1^{\text {st }}$ ed., Tomsk polytechnic university, Tomsk, 2009.

[26] DUNG, N. Q. and T. H. Q. MINH. "A Innovation Identification Approach of Control System by Fractional Transfer," IAES Indonesian Journal of Electrical Engineering and Computer Science, vol. 3, iss. 3, pp. 336-342, 2016.

\section{About Authors}

Quang Dung NGUYEN was born in Vietnam. He received his B.Sc and M.Sc. from Tomsk Polytechnic University, Russia in 2010 and 2012, respectively. His research interests include identification of control system. Fractional order system, distributed parameter system, renewable energy, SCADA systems and industrial communication network. He is working as Lecturer in Faculty of Electrical and Electronics Engineering, Ton Duc Thang University, Ho Chi Minh City, Vietnam.

"This is an Open Access article distributed under the terms of the Creative Commons Attribution License, which permits unrestricted use, distribution, and reproduction in any medium, provided the original work is properly cited (CC BY 4.0)." 